\title{
LE CONSEIL D'ETAT
}

Prof. Dr. Bernard PACTEAU*

On a commencé de célébrer en France le $2^{\circ}$ centenaire du Conseil d'Etat. Les français aiment les anniversaires.

Le Conseil d'Etat a été créé lui-même en 1799 par Napoléon.

C'est certainement l'un dés plus importants héritages de cette période. C'est aussi une des institutions françaises les plus solides.

Depuis 200 ans, la France a connu en effet plusieurs constitutions, avec des rćgimes politiques très divers. La vic politique française a été très instable. Notre organisation administrative a en revanche connu une très grande stabilité, et le Conseil d'Etat a toujours subsisté.

Le premier caractère du Conseil d'Etat français est donc cette ancienneté. Certains historiens ont même montré que le Conseil d'Etat puisait ses origines du temps de la monarchie d'avant la Révolution. Il existait effectivement alors près du Roi ce qu'on appelait un Conseil d'Etat participant à ses différentes fonctions d'administration et de justice.

Mais si le Conseil d'Etat est ainsi très ancien, il a aussi beaucoup changé.

En réalité, il a à la fois beaucoup changé et conservé son originalité .

C'est d'ailleurs sa raison d'exister. Ce qui justifie le Conscil d'Etat c'est à la fois qu'il a évolué et qu'il a gardé des caractères très particuliers qui le distinguent des autres tribunaux.

Ce sont ces deux aspects que je voudrais étudier devant vous. Je souhaite ainsi vous montrer à la fois l'évolution, les transformations et les améliorations progressives

\footnotetext{
* Université Montesquicu Bordcaux IV-France
} 
de cette institution et ses particularismes qui étonnent parfois et qui sont pourtant sa raison d'être.

\section{Les évolutions et transformations du Conseil d'Etat}

Il faut en effet se souvenir que le Conseil d'Etat est non pas un héritage de l'histoire, mais plutôt une création historique prógressive.

Il apparaît aujourd'hui au service des droits individuels.

Quand il a été institué, c'était au contraire dans un objectif très autoritaire.

Il fut créé en réalité non pas pour qu'il existe des juges particuliers sur les litiges administratifs mais pour çu'il n'existe aucun contrôle juridictionnel sur l'action administrative.

Cela nous conduit à voir, la manière très indirecte dont notre juridiction administrative s'est peu à peu forméc.

A l'époque de la grande Révolution de 1789, il avait été affirmé avant tout une interdiction pour les tribunaux judiciaires de «troubler de quelque manière que ce soit les opérations des corps administratifs» (loi des 16-24 ao`t 1790), et de «connaître des actes d'administration de quelque: nature qu'ils soient» (Décret du 16 fructidor an 3 - juin 1795).

C'est ce qu'on appelle la séparation des autorités administratives et judiciaires dont était de faire barrage au contrôle de l'action administrative par les tribunaux judiciaires ordinaires

Cette séparation a certes anciennement comporté ct comporte encore aujourd'hui quelques atténuations et aménagements.

Certains contentieux administratifs sont ainsi attribués aux tribunaux judiciaires,

- soit qu'ils ne mettent pas directement en cause des intérêts publics majeurs (par exemple : à l'égard des activités industriclles et commerciales des personnes publiques),

- soit qu'ils intéressent la sauvegarde essentielle des droits fondamentaux, l'autorité judiciaire étant considérée traditionnellement en France comme la "gardienne» de la liberté individuelle (ainsi que le redit la constitution du 4 octobre 1958 en son article 66).

Les juges rẹ́pressifs sont en outre investis du pouvoir d'écarter (par voie d'exception) l'application des actes administratifs qu'ils estiment illégaux, ainsi que la jurisprudence l'a reconnu et comme c'est désormais prévu expressément à l'article 111-5 du nouveau code pénal (entré en vigueur en 1994), à l'égard des actes «réglementaires ou individuels»: 
Le principe premicr et toujours primordial de notre système juridique demeure bien tout de même celui de l'incompétence et de l'impuissance des tribunaux ordinaires à l'égard du contenticux administratif.

Cette loi de 1790 est ainsi toujours en vigueur et elle cst toujours visée par les décisions des tribunaux.

Mais si le contentieux administratif retiré alors de la compétence des tribunaux judiciaires ne fut cependant pas confié à une «juridiction administrative».

A l'époque de la Révolution française, le contentieux administratif fut ainsi attribué non pas à d'autres juges mais à personne ou plutôt : aux autorités administratives elles-mêmes sclon le système de l'administrateur-juge.

C'était grave, c'était dangereux, c'était insatisfaisant.

Il faut ajouter que c'était voulu, c'était volontaire.

Tout un courant politique soutenait en effet la thèse selon laquelle il était convenable que l'administration soit indépendante des tribunaux, car, disait-on: juger l'administration, c'est encore administrer.

On invoquait même ici la grande théorie de la séparation des pouvoirs de Montesquieu. Et on défendait que si les tribunaux sont indépendants du législateur et de l'Exécutif, de la même manière le législateur et aussi les administrateurs doivent échapper au contrôle des juges.

Selon cette doctrine, le pouvoir des juges devait se limiter aux procès d'ordre civil entre personnes privées et à la sanction des infractions pénales.

Le contentieux administratif français naissant était bien alors séparé des juges judiciaires, il ne l'était pas de l'administration

Quand le Conseil d'Etat fut créé en 1799, il n'était pas d'ailleurs lui-même une juridiction. C'élait un organe purement administratif. sans aucune indépendance.

* D'une part, il dépendait entièrement du gouvernement pour ce qui est de son recrutement et de son organisation.

* D'autre part, il ne prenait lui-même aucune décision. Il se bornait à donner des avis, soit sur les projets de loi ou de décret qu'on lui soumcttait, soit aussi sur les réclamations des citoyens pour lesquels il pouvait seulement proposer une solution. C'est pourquoi on parlait alors de $\nmid j u s t i c e$ retenue.

Encore une fois, tout cela était voulu. On redoutait le contrôle de véritables juges sur l'action administrative. On redoutait les juges trop puissants. On préférait encore pas de juge ou des juges soumis (ce qui est la même chose). 
Naturellement, les auteurs soucieux des droits individuels et de respect de la légalité avaient critiqué ce système.

Une évolution devait justement avoir lieu:

Tout d'abord, dans la pratique, le Conseil d'Etat a très tôt commencé à se comporter en juridiction, avec une procédure d'examen des affaires proche de celle des tribunaux ; dans les affaire: contentieuses ses avis étaient toujours suivis. Un début de contrôle put ainsi avoir lieu sur les actions publiques. égards :

Puis, le Conseil d'Etat a effectivement obtenu son indépendance, et cela à deux

- d'une part, il fut créé un concours pour accéder aux premiers grades du Conseil d'Etat,

- d'autre part, une loi de 1872 établit que le Conseil d'Etat statuerait souverainement sur les procès qui lui étaicnt soumis, ce qu'on a appelé la «justice déléguée».

Grâce à ces bases nouvelles, et grâce aussi à des circonstances politiques très favorables à la défense des droits individuels, le Conseil d'Etat français a pu ainsi devenir l'instrument le plus puissant de soumission de l'action administrative à la légalité.

Depuis cent ans, le Conseil d'Etat a fait progresser le droit public français de deux grandes façons :

- le Conseil d'Etat a réalisé un contrôle de plus en plus important sur l'action publique, en matière de légalité comme en matière de responsabilité,

- Et il a aussi participé à la progression du droit public en imposant à l'administration des principes généraux qui n'étaient pas inscrits expressément dans les textes.

\section{Bien entendu, l'oeuvr' réalisée comporte des imperfections.}

On a surtout formulé deux grands reproches à la justice administrative française:

- le juge intervient trop tard, alors que l'action ou la décision administratives ont eu tous lcurs effets,

- le juge n'a pas les moyens de contraindre les autorités administratives à exécuter leurs obligations, c'est-à-dire que le juge dit le droit mais qu'il ne peut l'imposer vraiment et concrètement.

Il faut cependant observer que ces reproches sont de moins en moins justifiés. Des lois sont ainsi intervenues pour permettre plus efficacement au juge d'intervenir préventivement, et aussi d'adresser des injonctions à l'administration en vue de l'exécution de ses obligations. 
C'est aussi la preuve que le droit administratif français est toujours en transformation. C'est un vrai chantier. Et certainement, la qualité de ce droit administratif doit beaucoup au Conseil d'Etat. départ.

Tel a été le résultat de l'évolution. Tel n'avait cependant pas été son point de

On notera même que très curieusement le Conseil d'Etat avait été cré́ pour protéger l'administration et que bien au contraire il a exercé sur l'action administrative un contrôle très sévère, très fort et plus puissant certainement que celui auquel il devait faire échapper.

Le Conseil d'Etat a si bien oeuvré qu'il a été saisi de plus en plus souvent par les particuliers. Il en est résulté un encombrement.

Jusqu'en 1950, il était en réalité le seul tribunal administratif en France et pour presque tous les litiges administratifs. Longtemps, cela a suffi, mais après 1945 les recours sont devenus trop nombreux pour lui. Il recevait environ 6000 procès par an mais ne rendait que 4000 décisions.

On a donc alors institué des tribunaux de premier ressort qui sont désormais 35 , et le Conseil d'Etat devenait seulement juge d'appel.

Cette réforme a connu un grand succès. Elle a permis de faire face au froces cxmais elle a aussi encouragé la progression des procès. Ces tribunaux régionaux reçoivent ainsi aujourd'hui plus de 100000 procès par an. Ce succès a été tel que depuis 1980 le Conseil d'Etat a fini par être encombré cette fois comme juge d'appel.

C'est pourquoi on a aussi crế en 19875 cours administratives d'appel, qui sont désormais 6 et bientôt 7 ... et le Conseil d'Etat n'est plus alors que juge de cassation.

Il faudrait seulement dire que certains recours ne peuvent toujours être adressés qu'au Conseil d'Etat lui-même, comme par exemple les recours contre les décrets du gouvemement, et aussi que certains procès en appel lui sont réservés de façon à ce qu'ils soient jugés plus vite, comme en matière électorale.

Même si le Conseil d'Etat a $d^{\bullet}$ partager sa fonction avec d'autres tribunaux, il demeure bien naturellement le juge administratif principal. C'est lui qui fixe la jurisprudence et il est très soucieux de son unité. Il demeure aussi le modèle ; les nouveaux tribunaux et cours ont en effet été créés un peu de la même manière que lui, selon le même esprit à la fois d'indépendance vis-à-vis du système judiciaire et d'indépendance vis-à-vis du gouvernement.

La juridictionnelle française s'est ainsi progressivement consolidée.

Et elle a êté aujourd'hui dotée de bases juridiques constitutionnelles. 
Le Conseil constitutionnel a ainsi reconnu valeur constitutionnelle à la fois à lindépendance des juridictions administratives (Décision du 22 juillet 1980) et à leur compétence de principe envers les actes de la puissance publique (Décision du 23 janv. 1987).

La juridiction administrative ne doit donc plus être considérée comme une juridiction d'exception, mais comme la juridiction de droit commun du contentieux administratif.

Sans nul doute, c'était la solution la plus rationnelle.

L'amdinistrateur-juge était inacceptable.

Mais les procès administratifs ne sont certainement pas de la même nature que les procès civils; ils ne mettent pas en cause les mêmes intérêts. Dès l'époque de la révolution française, on avait cu l'intuition de cette spécificité, mais évidemment, on était allé trop loin.

- Il convenait certainement de retirer le contenticux administratif aux tribunaux judiciaires ordinaires, mais il convenait aussi de confier ce contentieux à de véritables juges.

Cette dualité de tribunaux cst égalitaire: aucune des deux juridictions n'a autorité sur l'autre; aucune juridiction suprême n'a non plus autorité sur elles.

Pour régler les difficultés nés de la dualité juridictionnelle, il y a tout de même un Tribunal des conflits composé paritairement de membres du Conseil d'Etat et de la Cour de cassation et qui est placé sous l'étonnante présidence du ministre de la justice, mais qui n'intervient qu'en cas de partage de voix, ce qui est très rare: environ 10 cas depuis 1872 ; un nouveau cas s'est cependant présenté en mai 1997 avec alors une résurgence des débats sur la légitimité de pareille présidence.

Le Tribunal de conflits n'a que des compétences d'attuibution, mais qui ont connu historiquement de l'expansion et qui lui ont ainsi permis de devenir vraiment l'arbitre de la dualité juridictionnelle en France.

Le Tribunal des conflits a une activité quantitativement réduite (environ 50 affaires par an) mais toujours déterminante qualitativement, non sculement pour garantir mais aussi souvent pour définir la répartition des compétences respectives des deux ordres en l'absence de textes ou de textes suffisamment précis.

Le système des "corflits» avait d'abord été aménagé avant tout pour empêcher les tribunaux judiciaires de connaître de procès administratifs. Cest toujours une des fonctions du tribunal des conflits et qui est mise en ocuvre sur intervention des autorités publiques (on parle alors de conflit positif d'attribution), en notant qu'il n'existe pas de procédure permettant d'empêcher inversement un tribunal administratif de déborder de sa propre compćtence vers le domaine judiciaire. 
Plus largement, le tribunal des conflits est aujourd'hui placé au service des juges et des justiciables pour leur éviter de s'égarer dans le choix du tribunal compétent.

Ses moyens d'intervention ont d'ailleurs été plusieurs fois élargis et améliorés, de façon en particulier à mieux combattre l'hypothèse du «conflit négatif d'attribution», c'est-à-dire l'hypothèse o o aucun tribunal, ni judiciaire ni administratif, ne voudrait se reconnaître compétent sur un procès qui relève bien cependant de l'un ou de l'autre.

Un décret de 1960 a permis aussi au Conseil d'Etat et à la Cour de cassation de renvoyer spontanément toute question sérieuse de compétence soulevée devant eux. contestce.

L'existence de la juridiction administrative et du Conseil d'Etat est encore parfois

\section{On entend à cet égard deux critiques :}

- le système de dualité est gênant pour les justiciables dès lors qu'il y a hésitations ou erreurs dans le choix du tribunal compétent.

Ces hésitations et erreurs ne sont cependant pas très nombreux et le mécanisme du tribunal des conflits permet convenablement d'y faire face.

- le système de dualité n'a plus de raison d'être aujourd'hui, en somme, il est inutile: puisque tout le monde est d'accord pour réaliser un contrôle sur l' administration, pourquoi ne pas rendre el restituer ce contrôle aux juges ordinaires?

Mais c'est oublier que la nécessité subsiste d'un régime spécial du contentieux administratif et que les tribunaux administratifs ont justement la grande qualité d'être constitués de façon à bien garantir cette mission.

Et cela me conduit à parler des caractéristiques particulières que conserve toujours la justice administrative en France et plus précisément le Conseil d'Etat .

\section{Chez le Conseil d'Etat français, il y a certes eu rupture.}

La jurisprudence d'aujourd'hui, l'image actuelle du Conseil d'Etat sont foncièrement différents de ce qu'elles étaient il y a seulement cent ans alors que le pouvoir discrétionnaire était triomphant, que la responsabilité publique était quasiment inexistante.

\section{Mais il y a aussi une grande continuité.}

Si le Conseil d'Etat a avancé, progressé, c'est ains̀i tout en conservant du passé certains traits originaux hérités du passé, mais en réalité soigneusement préservés et parfois même développés et qui interdisent d'y voir pleinement et totalement une juridiction, en tout cas pas une juridiction comme les autres. 
C'est ce qui fait dire que le Conseil d'Etat français constitue un curieux mélange de tradition et de modernité.

C'est une institulion qui s'est toujours ouverte au changement mais qui est également toujours restée attachée à ses racines et à ses liens avec les autorités administratives dont il est le contrôleur.

Il en résulte assurément des caractères qui peuvent étonner.

Cela oblige en tout cas d̀ regarder le Conseil d'Etat avec attention et avec réalisme.

\section{Les caractères originaux conservés par le Conseil-d'Etat.}

La juridiction administrative demeure régulièrement critiquée et dénoncée en France au motif qu'elle présenterait moins de garanties que la justice judiciaire de droit commun et qu'en particulier elle serait plus proche de l'administration, et de ses préoccupations, que de la juridiction.

Assurément, l'organisation de la juridiction administrative présente des caractères qui étonnent et qui peuvent même heurter, s'agissant en particulier de son instance supérieure, le Conseil dTEtat.

\section{Le Conseil d'Etat n'a ainsi à sa tête qu'un vice-président,}

son président de droit demeurant le chef du gouvernement.

Cette présidence par le chef du gouvemement est purement protocolaire.

Cela constitue tout de même une situation curieuse.

Ou bien la présicence du Premier ministre est purement apparente, et il faudrait donc supprimer cette ambiguÔté. Ou bien elle est effective et il faut la supprimer comme constituant une anomalic.

Par deux fois récemment, des personnes ont d'ailleurs tout de même réclamé la démission du Premier ministre de son titre de président du Conseil d'Etat ...

Et un recours a été intenté devant le Conseil d'Etat lui-même contre le refus alors exprimé par le Premier ministre face à cette demande. La question n'était pas forcément facile à traiter ... mais lo: Conseil d'Etat a pu juger que cette présidence était prévue par une loi et donc que le recours de ces personnes était l'équivalent d'un recours contre le refus du Premier ministre de déposer un projet visant à l'abrogation de cette loi, recours qui échappe par nature au contenticux administratif ...

On pourrait évidemment donner au Conseil d'Etat une présidence totalement autonome. Il est probable que le Conseil d'Etat lui-même ne le souhaite pas, sans doute parce que c'est une tradition, également parce que c'est pas gênant pour lui, car cette présidence du chef du gouvernement est en fait pour lui un élément de prestige en le rattachant à l'Etat lui-même. 
C'est également un indice qui marque que le Conseil d'Etat n'est pas considéré en France comme un tribunal; il a des fonctions de tribunal, mais d'autres aussi, et une nature spéciale, sui generis.

Il existe toujours de larges possibilités de nomination discrétionnaire en son sein par ce qu'on appelle le tour extérieur.

Ce tour extérieur ne peut concerner les auditeurs mais en revanche $1 / 4$ des emplois de maître des requêtes et $1 / 3$ des conseillers $d$ tEat avec une seule condition :avoir 45 ans).

Naturellement les nominations opérées donnent toujours lieu à débat.

Il faut cependant observer :

- que les conseillers nommés au tour extérieur sont ensuite soumis au même statut et aux mêmes exigences d'impartialité que les autres membres du Conseil d'Ẹtat,

- que le travail très collégial au sein du Conseil d'Etat fait qu'ils ne sauraient avoir une influence particulière,

- que le Président de la République n'en a jamais usé d'une façon abusive quant à la compétence et aux expériences ou à la personne de ceux qui entrent ainsi au Conseil d'Etat par le "tout extérieur».

Une loi de 1994 a en outre prévu que toute nomination au tour extérieur serait précédée d'un avis - certes purement consultatif - du vice-président du Conseil d'Etat.

Un contentieux pourrait aussi avoir lieu sur ses nominations et conduirait le Conseil d'Etat à vérifier si la nomination opérée n'est pas entachée au moins d'une «erreur manifeste» quant aux compétences et qualités de l'intéressé.

Dans d'autres domaines, il est d'ailleurs déjà arrivé que le Conseil d'Etat annule une nomination au tour extérieur.

En réalité, ce tour extéricur est ressenti en France comme une chance et une richesse autant que comme un danger. Il s'inscrit dans un esprit d'ouverture du Conseil d'Etat sur le monde qui l'entoure, et comme un élément de diversification de son recrutement.

Encore faut-il se souvenir que les membres du Conseil d'Etat n'ont pas un statut de magistrat; ils n'ont pas officiellement l'inamovibilité et leur statut n'est pas celui prévu par la constitution pour les membres de l'autorité judiciaire.

L'originalité du Conseil d'Etat français par rapport aux juridictions habituelles mais non par rapport à d'autres Conseil d'Etat - est d'autre part et surtout d'exercer des fonctions à la fois juridictionnelles et consultatives;

Là aussi c'est un héritage. 
A sa création, le Conscil d'Etat était même principalement chargé de rédiger les projets de loi ainsi que les règlements pubfics. Il était avant tout conseiller et accessoirement juge, très accessoirement même car il avait peu d'activité contentieuse et que même à cet égard il ne faisait que proposer des projets d'arrêts au chef de l'Etat.

Aujourd'hui, le Conseil d'Etat est surtout connu de l'extérieur comme juge.

Il demeure tout de même donneur d'avis.

Depuis 1945 et surtout depuis 1958, et même encore plus récemment cette fonction a d'ailleurs progressé alors qu'elle avait plutôt décliné à l'époque de la III ${ }^{\circ}$ République d'esprit et de pratique très parlementaire et donc soucieuse de réserver les pouvoirs au Parlement et au Gouvernement qui en dépend.

Il a en particulier été consulté sur le projet de constitution de la $\mathrm{V}^{\circ}$ République.

Le Conseil d'Etat est ainsi consulté obligatoirement :

- sur les projets de loi,

- et sur de nombreux projets de décret. Les lois sont ainsi nombreuses à prévoir que telles ou telles mesures devront être prises par décrets en Conseil d'Etat. La constitution elle-même prévoit la consultation du Conseil d'Etat quand le gouvemement veut modifier par décret d'un loi antérieure à la constitution de 1958 mais prises dans une matière qui est aujourd'hui réglementaire.

Sa consultation est également parfois imposée pour des actes plus particuliers par exemple pour certaines expropriations, ou l'autorisation de certains travaux particulièrement importants, et aussi l'attribution aux associations de la qualité d'associations "reconnues d'utilité publique», ce qui élargit leurs droits et leurs moyens financiers.

Le Conseil d'Etat a donné en 1996 des avis sur 1400 textes dont :

105 sur des projets de lois ou d'ordonnances

485 sur des décrets réglementaires

772 sur des décrets ou arrêtés individucls,

+ environ 500 textes communautaires en vue de déterminer s'ils comportaient des dispositions de nature législative en droit français.

Souvent et même de plus en plus, le gouvernement lui demande aussi des avis sur des points de droit particulier et généralement très controversées, comme par exemple en 1989 sur le point de savoir si les directeurs d'école pouvaient refuser que des jeunes filles islamistes portent un voile à l'intérieur de l'école et refusent de participer à certaines activités scolaires. 
Des études peuvent aussi lui être demandées, et le sont très régulièrement sur des sujets parfois très délicats, telles que l'éthique en matière de recherche biologique et médicale, la responsabilité des agents publics, l'informatique.

La vérité c'est donc que ces activités non juridictionnelles ont été non seulement conservées mais encore qu'elles ont été développées à l'époque actuelle.

Le gouvernement y est très attaché.

Il a même souhaité que ces deux fonctions soient le moins possible dissociées.

Il a été ainsi prévu depuis 1963 sur chaque membre du Conseil d'Etat devrait autant que possible participer à la fois à une section administrative et à la section du contenticux, ce qu'on appelle «la double affectation» qui est même de droit pour les auditeurs et les maîtres des requêtes, et en outre les formations de jugement associent de façon systématique des conseillers en provenance de chacune des catégories de section.

Par là le Conseil d'Etat participe en effet à la création même du droit. Cela lui donne aussi une source d'information. Et assurément, c'est un travail juridique passionnant que d'analyser un projet, d'en rechercher les imperfections ou les différentes implications. Le Conseil d'Etat ne se contente pas d'ailleurs d'exprimer un avis mais rédige lui-même un contre-projet.

Si le Conseil d'Etat a ainsi une double fonction, les autres juridictions administratives elles aussi participent à l'action publique même si c'est avec moins de compétences el si c'est avec des compétences moins prestigieuses puisqu'exercées auprès des autorités publiques territoriales.

En 1996, l'ensemble des tribunaux administratifs avaient reçu seulement 164 demandes d'avis des préfets (171 en 1995, 206 en 1994).

En revanche, les conscillers des tribunaux administratifs et des cours administratives d'appel participent à de très nombreuses commissions administratives de recours et décisionnelles (commissions d'expulsions, commissions fiscales, commissions élcctorales); il y a même une tendance peut-être exagérée à faire appel à eux pour présider de nombreuses instances publiques, ce qui rend hommage à leur compétence mais qui affecte le bon fonctionnement des juridictions.

Evidemment, cette dualiét de fonctions alourdit le travail des juges administratifs et certainement ccla contribue à retarder la solution des procès ....

Mais encore une fois, le système français a préféré remodeler le système des juridictions de façon à préserver cette dualité de fonctions.

Si aujourd'hui, cette dualité est à nouveau discutée, c'est qu'on s'interroge sur son bien-fondé, voire sur ses dangers ...

LE DEBAT SUR CES FONCTIONS ADMINISTRATIVES OU NONJURIDICTIONNELLES DU CONSEIL D'ETAT est lui-même ancien, mais il se 
renouvelle aujourd'hui à la cause d'un arrêt rendu par la cour européenne des droits de l'homme en 1995 dans une affaire Procola qui a concerné le Conseil d'Etat du Luxembourg mais qui intéresse aussi d'autres Conseils d'Etat ... et notamment celui de France.

\section{Tout d'abord, est-ce bon pour l' administration ?}

Je veux dire: est-ce bón pour la qualité de l'action administrative, est-ce que cela contribue à améliorer la qualité des décisions publiques?

L'influence des avis et études du Conseil d'Etat est naturellement difficile à mesurer.

Assurément ces avis ne sont pas toujours suivis.

S'agissant des projets de loi, lavis du Conseil d'Etat intervient d'ailleurs alors que le débat parlementaire n'est pas commencé. Inversement, s'agissant des décrets il intervient tard à un moment $0^{-}$la plupart des arbitrages ont déjà été décidés.

On observe aussi que le poids de ces avis peut être affaibli par leur caractère confidentiel; le principe est en effet que ces avis sont secrets, seul le gouvernement peut décider de les rendre publics.

Les observateurs de la vie administrative sont tout de même unanimes à conclure à l'utilité de ces consultations du Conseil d'Etat, comme d'ailleurs à l'utilité de la participation de magistrats administratifs à différentes instances et commissions publiques.

\section{Leur valeur est avant tout technique.}

Ils contribuent à améliorer la rédaction même des textes administratifs et des projets de loi. Ils mettent aussi en évidence les imperfections juridiques, et cela aussi bien au travers des irrégularités que des incohérences.

Par exemple, le Conseil d'Etat mettra en évidence le manque de coordination entre deux textes, ou les difficultés qu'une réforme ponctuelle aura à se combiner avec les règles générales de notre administration.

Naturellement, celui-ci s'abstient de toute appréciation politique. Simplement, le Conseil d'Etat peut exprimer une appréciation d'opportunité quant à l'intérêt ou les dangers sociaux du projet qui lui est présenté.

Il cherche en tout cas toujours à mettre en évidence les difficultés de mise en oeuvre de telle ou telle disposition.

Il recherche aussi la compatibilité du texte proposé aussi bien avec la constitution qu'avec nos engagements internationaux.

En pratique, les avis du Conseil d'Etat sont donc écoutés, et souvent aussi suivis, parce que c'est l'intérêt même de l'administration. 
Le Conseil d'Etat est en tout cas très soucieux de vérifier que sa consultation a bien eu lieu effectivement et qu'elle a eu lieu régulièrement.

Récemment le Conseil d'Etat a ainsi annulé un décret décidant le changement de nom d'une commune après avoir constaté que le gouvernement avait oublié de lui soumeure ce changement pour avis.

Une autre fois, il avait pu établir que le décret avait été signé avant que son avis ait été communiqué et transmis au gouvernement. Un changement de circonstances depuis la consultation opérée obligerait aussi le gouvernement à la répéter ainsi quil en va dans le droit commun des consultations administratives.

Le caractère non public des avis du Conseil d'Etat donne lieu à débats: aux yeux de l'opinion, il devient un peu mystéricux et on se demande si son secret ne signifie pas qu'il est indigne d'être connu; d'autre part, ce secret favorise les rumeurs et indiscrétions qui peuvent avoir des inconvénients plus graves encore.

Inversement, la tendance générale est de considérer que ce secret favorise la liberté et la sérénité du travail du Conseil d'Etat, que cela évite au Conseil d'Etat d'être placé au coeur d'un débat politique et partisan car le sens de son avis et aussi ses arguments seraient désormais directement utilisés. Il est d'ailleurs de règle que les rapporteurs des commissions des assemblées parlementaires peuvent se faire communiquer ses avis par le gouvernement.

On en vient à la seconde question :

Cette dualité de fonctions, cette participation à l'action et aux décisions publiques, est-ce bon pour le juge? est-ce bon pour la juridiction ? Est-ce bon pour la justice?

Le juge risquerait d'être trop proche des intérêts administratifs, serait trop proche aussi des gouvernants et des autorités administratives.

Ce pourrait - là aussi - être une faiblesse.

La cour européenne des droits de l'homme y vu aussi une possible atteinte à l'impartialité des juridictions si les personnes qui ont donné un avis favorable sur un texte sont ensuite chargées de le juger .

L'impartialité est certes la première des garanties des justiciables. C'est aussi la première loi des magistrats. L'indépendance même des magistrats a pour fonction de garantir ceute impartialité.

Ou bien: on peut dire aussi que l'impartialité est la forme suprême de l'indépendance du magistrat; elle signifie son indépendance non seulement vis-à-vis des autres pouvoirs, mais aussi de lui-même, de ses passions et de ses préférences personnelles. 
Dans cette affaire P'rocola, la cour a d'ailleurs défendu une doctrine très rigoureuse de l'impartialite.

Selon elle, même si les juges sont parfaitement impartiaux, il ne faut pas que l'organisation même du tribunal crée un doute chez les justiciables.

C'est vrai, cela conduit à privilégier les «apparences». Il ne s'agit certes pas de se satisfaire de l'apparence de l'impartialité au détriment de la réalité, mais d'exiger EN PLUS de l'impartialité effective l'image même de l'impartialité, et donc de condamner un jugement rendu alors que les apparences de l'impartialité n'étaient pas réunies même si cela n'a eu aucune conséquence concrète. d'inspirer.

C'est juste et cela s'inscrit dans la confiance que les juridictions se doivent

On ne peut donc que souscrire à la phrase centrale de l'arrêt PROCOLA :

*Un simple doute, aussi peu justifié soit-il, suffit à altérer l'impartialité du tribunal .....

C'est à cela que répondent les règles générales de récusation qui sont aussi pratiqués dans la justice administrative en France s'il apparaît qu'un juge qui pour des raisons tenant à ses intérêts, ses parentés ou ses amitiés ne présenterait pas toutes les garanties d'impartialité.

Le droit de la procédure pénale est naturellement très strict aussi en ce domaine.

\section{La question est alors de savoir si ces principes condamnent}

\section{la dualité de fonctions du Conseil d'Etat français.}

Je pense que non.

La situation de l'affaire PROCOLA était tout à fait particulière.

Il s'agissait d'un Conseil d'Etat peu nombreux (au total : 21 membres) et dans lequel il y avait presque identité totale entre la formation qui avait formulé l'avis et celle qui devait rendre l'arrêt ( 4 sur 5 des membres du comité du contentieux). L'arrêt insiste d'ailleurs sur toutes ces caractéristiques.

Mais il ne faut pas en étendre exagérément les implications, s'agissant de la France ou d'autres pays :

D'une part, l'arrêt PROCOLA n'a pas interdit radicalement à un même organisme d'exercer des fonctions à la fois consultatives et juridictionnelles.

Il en ressort seulement que POUR UNE MEME ET UNIQUE DECISION, il ne doit pas y avoir identité des deux formations appelées à intervenir et qu'en tout cas la formation contentieuse ne soit pas quasi-uniquement composée de personnes ayant antéricurement exprimé un avis favorable à la mesure en cause. 
D'autre part, si le droit français mêle effectivement les activités administratives et contentieuses du Conseil d'Etat c'est tout de même et en revanche en les dissociant netuement les modalités d'exercice.

On retrouve souvent les mêmes personnes dans les instances d'avis et dans les instances d'arrêt, mais ces formations sont quand même toujours différentes.

Les procédures sont également totalement différenciées.

Tout est ainsi mis en oeuvre pour garantir la spécificité de l'instance juridictionnelle èt sa pleine indépendance vis-à-vis de l'instance consultative antérieure.

Il est d'ailleurs arrivé que le Conseil d'Etat censure et condamné comme juge ce qu'il avait accepté comme conseiller .... C'est là une hypothèse qui rassure mais qui inquiète aussi .... en faisant douter de la solidité de notre droit public. Mais il peut se faire que gr, ce à un examen contentieux plus approfondi et davantage contradictoire une irrégularité apparaisse. On a pu dire qu'il y avait eu en somme appel au Conseil d'Etat «mieux informé».

Encore faudrait-il rappeler que conseiller le gouvernement ne signifie nullement être son allié, son auxiliaire.

Le Conseil d'Etat n'est pas - ou en tout cas n'est plus depuis bien longtemps un conseiller du gouvemement qui juge aussi, mais bien davantage un juge qui en toute donne des avis - plus d'ailleurs que des "conseils" - à l'Exécutif. Et ces avis les donne avec la même indépendance que celle avec laquelle il rend ses arrêts.

Il est vrai assurément qu'une dissociation des instances consultatives et juridictionnelles doit être assurée.

Certainement, il faut aussi éviter que participe au jugement une personne dont l'avis aurait été exprimé publiquement ou dont l'avis serait nécessairement connu de l'administration si cet avis avait été rendu individucllement.

Mais je ne pense pas que lc Conscil d'Etat français soit contestable au nom de la jurisprudence PROCOLA, ni que cette jurisprudence condamne par principe la dualité des fonctions consultatives et juridictionnelles

L'opinion doctrinale dominante en France considère que cette dualité de fonctions est sans doute un héritage du passé, mais que c'est aussi une chance.

Il a été très souvent constaté que le juge administratif, parce que plus proche de la vie administrative, parce qu'il la connaît mieux, peut davantage encore que le juge judiciaire élaborer un droit bien adapté aux exigences de la vie publique, et un droit qui d'ailleurs n'est pas nécessairement plus complaisant envers l'administration. 
Cette originalité du juge administratif est en somme devenue sa raison d'être: la dualité juridictionnelle ne se justifie que si les juges administratifs sont des juges différents.

Elle est aussi devenue très largement sa force. C'est parce qu'il connaît mieux l'administration que le juge administratif a souvent pu élaborer des principes et des tochniques de contrôle plus sévères.

On dit parfois le droit administratif est un miracle. On pourrait dire que le juge administratif est lui-même un mystère. 